\title{
Repurposing Therapeutics for the Wuhan Coronavirus nCov-2019: Supercomputer-Based Docking to the Viral S protein and Human ACE2 Interface
}

\author{
Micholas D. Smith ${ }^{1,2}$ and Jeremy C. Smith ${ }^{1,2,3^{*}}$ \\ * Corresponding author: smithjc@ornl.gov \\ ${ }^{1}$ Center for Molecular Biophysics, University of Tennessee/Oak Ridge National Laboratory, Oak Ridge National \\ Laboratory, P.O. Box 2008, Oak Ridge, TN 37831 \\ ${ }^{2}$ Department of Biochemistry and Cellular and Molecular Biology, University of Tennessee, M407 Walters Life \\ Sciences, 1414 Cumberland Avenue, Knoxville, TN 37996 \\ ${ }^{3}$ Oak Ridge National Laboratory, P.O. Box 2008, Oak Ridge, TN 37831
}

This manuscript has been authored by UT-Battelle, LLC under Contract No. DE-AC05-000R22725 with the U.S. Department of Energy. The United States Government retains and the publisher, by accepting the article for publication, acknowledges that the United States Government retains a non-exclusive, paid-up, irrevocable, world-wide license to publish or reproduce the published form of this manuscript, or allow others to do so, for United States Government purposes. The Department of Energy will provide public access to these results of federally sponsored research in accordance with the DOE Public Access Plan (http://energy.gov/downloads/doe-public-access-plan ). 


\begin{abstract}
The novel Wuhan coronavirus (nCov-2019) has been sequenced, and the virus shares substantial similarity with SARS-CoV. Here, using a computational model of the spike protein (S-protein) nCov-2019 interacting with the human ACE2 receptor, we make use of the world's most powerful supercomputer, SUMMIT, to enact an ensemble docking virtual high-throughput screening campaign and identify small-molecules which may be repurposed to disrupt host-virus interactions. A ranked list of compounds is given that can be tested experimentally.
\end{abstract}

\title{
Introduction
}

On the penultimate day of 2019, health officials at the Wuhan Municipal Health Commission (Hubei Province, China) reported an occurrence of concentrated pneumonia in the city of Wuhan ${ }^{1-2}$. Shortly after reporting the outbreak, the Chinese Center for Disease Control (Chinese CDC) and local Chinese health workers determined that the cause of the outbreak was a novel coronavirus denoted, initially, as Wuhan Cov (and now referred to as nCov-2019) ${ }^{3}$. By Jan. $10^{\text {th }}$, 2020 , the genome sequence of nCov-2019 was released ${ }^{4}$. On Jan. $20^{\text {th }}, 2020$, Xu et al ${ }^{2}$, compared the available sequences of those of other coronaviruses and demonstrated that nCov-2019 is within the SARS coronavirus (SARS-CoV) family.

Human host infections by SARS-CoV are known to associate with interactions between the viral spike protein (S-protein) and human host receptors. Using the genome data available, Xu et al. sought to investigate whether the $\mathrm{nCoV}-2019$ S-protein would have virial-host interactions similar to SARS-CoV. Using standard template-based modeling techniques, a 3D structural model of the nCoV-2019 S-protein interacting with the human ACE2 receptor (using the SARSCoV S-protein as modeling template) was generated. Binding affinity calculations using this 
structural model suggest that, indeed, nCoV-2019 and SARS-CoV may share a common hostreceptor: Angiotensin-converting enzyme $2(\mathrm{ACE} 2)^{2}$. Since the first report by Xu et al., additional work has also provided evidence of the nCoV-2019 S-protein binding to the ACE2 receptor $^{5-7}$.

The current model of the nCoV-2019 S-protein-human ACE2 complex facilitated infection suggests that a reasonable target for structure-based drug discovery might be to disrupt the viral S-protein-ACE2 interface. However, the development of novel therapeutics typically takes years. Given that the outbreak of nCoV-2019 may become a global challenge ${ }^{8}$, it would be of great benefit to identify and repurpose already well-characterized small-molecules and approved drugs for use in combating the disease. Here, combining restrained enhanced-sampling molecular dynamics simulations and small-molecule docking, we present twenty-seven currently available compounds that are predicted to bind within the nCoV-2019 S-protein-ACE2 interface and may serve to disrupt the complex and limit infection.

\section{Methods}

Ensemble docking is the strategy by which an ensemble of receptor (in this case, the nCoV-2019 S-protein-ACE2 interface) conformations is generated, typically through the use of molecular dynamics simulations and structural clustering, and used in place of a single receptor configuration during small-molecule docking calculations to account for receptor flexibility ${ }^{9-10}$. By accounting for the conformational diversity of the receptor ensemble docking enhances the likelihood of identifying predicted hits (enrichment), which may be lost when screening against a single configuration ${ }^{9}$. We have previously applied this technique to derive experimentallyverified hits for several protein targets ${ }^{11-19}$. Briefly, the work reported here was performed in 
three phases: structural modeling, molecular simulations (ensemble building), and smallmolecule docking (in silico ligand screening)

\section{$\underline{\text { Structural Modeling and Molecular Dynamics Preparation }}$}

Recent structural modeling work by Xu et al. suggested that the S-protein from nCoV-2019 has a strong binding affinity with the ACE2 receptor ${ }^{2}$. However, the coordinates of the structural model were not provided. Therefore, we generated a model of the nCoV-2019 S-protein-ACE2 complex (which we provide as supplementary material). Using SWISS-MODEL ${ }^{20-21}$, the

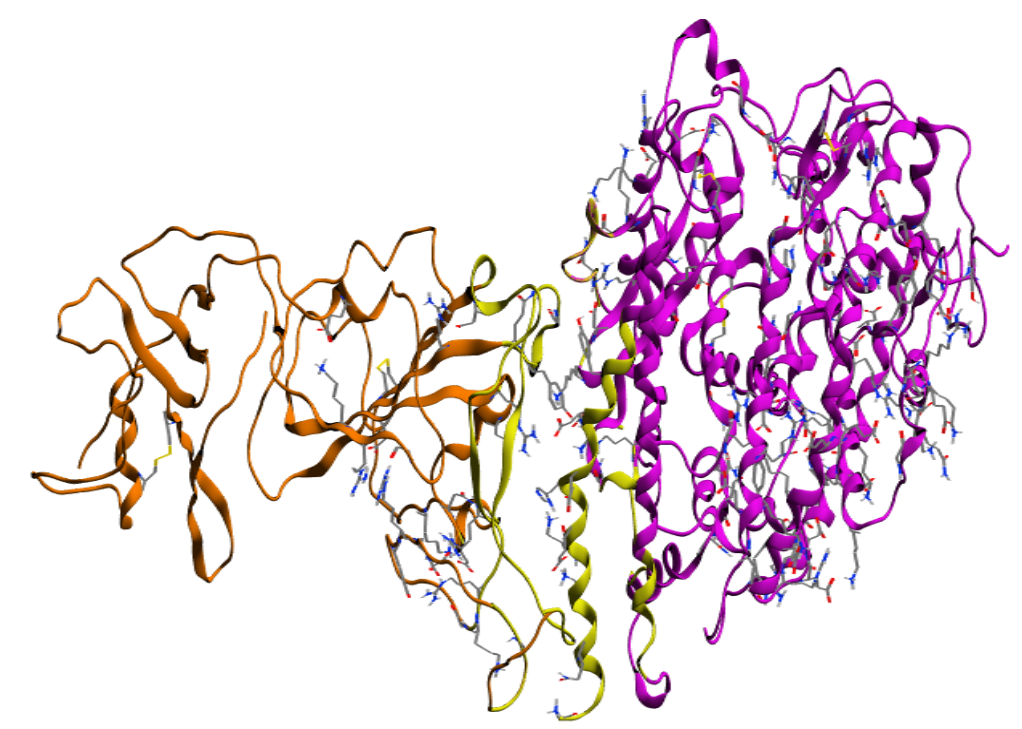

Figure 1) Rendering of nCoV-2019 S-protein and ACE2 receptor complex. Orange ribbons represent the S-protein, purple corresponds to ACE2, and yellow is a highlight of the interface targeted for docking.

sequences for the nCoV-2019 S-protein (NCBI Reference Sequence: YP_009724390.1) and ACE2 receptor were used along with the crystal structure of the SARS-CoV S-protein in complex with ACE2 (PDB: 2AJF), as a template, to generate a model of the nCoV-2019 S-protein and ACE2 receptor complex. A rendering of the complex is provided in Figure 1. 
To prepare the system for molecular dynamics simulations, the structural model was centered in a periodic simulation box of $9.895 \mathrm{~nm} \times 8.525 \mathrm{~nm} \times 17.415 \mathrm{~nm}$ and solvated in the (CHARMM) TIP3P water model ${ }^{22}$ using the gmx editconf and gmx solvate tools from the GROMACS molecular dynamics simulation suite ${ }^{23}$. As the total charge of the S-protein-receptor complex was non-zero, 21 sodium ions were added to neutralize the system. To remove potential clashes between the solvent, ions, and S-protein-receptor complex, energy minimization calculations (using GROMACS with the CHARMM36 force-field ${ }^{24}$ ) were performed with a convergence criterion of $1000 \mathrm{~kJ} / \mathrm{mol} \mathrm{nm}^{-1}$. Following the energy minimization, a short, 1ns NPT relaxation simulation was performed to relax the box dimensions. For the NPT simulation, the pressure and temperature were controlled using the Berendsen baro/thermostat ${ }^{25}$, and the simulation was performed using an integration time-step of $2 \mathrm{fs}$. For both NPT relaxation simulation and energy minimization calculations, short-range interactions were treated with a smooth force-switch cutoff of $1.2 \mathrm{~nm}$, and long-range electrostatics were treated using the PME (Particle-MeshEwald) formalism, as implemented within GROMACS ${ }^{26}$. For the relaxation simulation, hydrogen-bonds were restrained with the LINCS algorithm ${ }^{27-28}$.

\section{$\underline{\text { Restrained Temperature Replica-Exchange Molecular Dynamics }}$}

The critical requirement of ensemble docking is the generation of an ensemble that captures a wide array of the conformational space of the target receptor. Here we produced such an ensemble of receptor states by performing a temperature replica-exchange molecular dynamics simulation (T-REMD), with 46 replicates at temperatures from $310 \mathrm{~K}$ to $350 \mathrm{~K}$, of the S-proteinreceptor complex with residues more than $1.5 \mathrm{~nm}$ from the interface held fixed by harmonic restraints (with force-constants of $1000 \mathrm{~kJ} / \mathrm{mol} \mathrm{nm}^{-1}$ ). The T-REMD simulations generated trajectories that were $40 \mathrm{~ns}$ in length and the last $35 \mathrm{~ns}$ of each simulation used for analysis (35ns 
per replicate for a total of $1.61 \mu$ s of sampling). Simulations were performed using the GROMACS simulation suite (as compiled on the ORNL SUMMIT supercomputer) within the NPT ensemble with an integration time-step of $2 \mathrm{fs}$, a frame-saving rate of $10 \mathrm{ps}$, and with exchange attempts every 500ps (with an exchange rate of $\sim 20 \%$ obtained throughout the simulation). Short-range interactions and long-range electrostatics were computed using the same cutoffs as those used in the NPT relaxation simulations (see Structural Modeling and Molecular Dynamics Preparation). The temperature was maintained with the V-rescale thermostat ${ }^{29}$, while the pressure was maintained using the Berendsen barostat ${ }^{25}$. The Berendsen barostat was used to accommodate the use of position restraints within the simulation. As with the NPR relaxation, all hydrogen-bonds were subjected to constraints, using the LINCS algorithm.

Following the T-REMD simulation frames from all replicates (regardless of temperature) were concatenated into a single file and used for structural clustering. Root-mean-squared (RMSD) based structural clustering of the conformations defined by the heavy-atoms of each side-chain within the S-protein-receptor complex was performed with the gmx cluster tool of GROMACS using the GROMOS clustering algorithim ${ }^{30}$ and a cutoff of $0.2 \mathrm{~nm}$. Six clusters were identified via this clustering method and are provided in the Supplementary Material (SI) for interested readers.

After identifying the six clusters noted above, the interface region (see figure 1) of each structure was extracted and converted, using Autodock Tools ${ }^{31}$, into PDBQT formatted files for subsequent molecular docking calculations.

\section{Ligand Library Choice}


As the aim of this work was to identify previously approved small-molecules (along with metabolites and natural products) for repurposing in treating nCoV-2019 infection, we made use of the SWEETLEAD molecular library ${ }^{32}$. Each ligand within the SWEETLEAD library was converted from SDF format to the PDBQT format used in Autodock Vina (Vina) by first converting the SDF format to mol2 using MOE2016 $6^{33}$ and then Autodock tools ${ }^{31}$.

\section{$\underline{\text { in Silico Screening/Molecular Docking }}$}

Molecular docking was performed using a special POWER9 build of Vina ${ }^{34}$ for SUMMIT. Docking calculations were performed with a search box of $1.2 \mathrm{~nm} \times 1.2 \mathrm{~nm} \times 1 . \mathrm{x} 2 \mathrm{~nm}$ centered within the S-protein-ACE2 receptor interface and an exhaustiveness setting of 250. To allow for the rapid evaluation of the SWEETLEAD library, while also making use of high search exhaustiveness, docking calculations were performed in parallel using a generic script-launching MPI wrapper compatible with SUMMIT (provided in the SI for interested readers).

Of the 9127 ligands within the SWEETLEAD library, only a subset could be successfully scored with the search box restriction (see Table 1).

Table 1 Number of successful docking calculations for each receptor (cluster) conformation. The total number of docking calculations are also noted.

$\begin{array}{lllllll}\text { Cluster 0 } & \text { Cluster 1 } & \text { Cluster 2 } & \text { Cluster 3 } & \text { Cluster 4 } & \text { Cluster 5 } & \text { Total } \\ 8574 & 8587 & 8581 & 8578 & 8621 & 8634 & 51575\end{array}$

As a practical matter, a Vina score threshold was set to identify binding ligands. This threshold was set to $-7 \mathrm{kcal} / \mathrm{mol}$. After scoring each of the ligand-protein interface complex geometries generated during docking, the top four ligand-interface complexes were extracted for additional 
analysis using $\mathrm{MOE}^{33}$. For the top four ligand-interface complexes, energy minimization was performed in MOE with default convergence setting and with the MMF94x force-field ${ }^{35}$ to refine the ligand-interface. The refined structures were then used to generate $2 \mathrm{D}$ interaction maps between the ligand and the protein residues of the S-protein-ACE2 receptor interface.

\section{Results \& Discussion}

Of the 51575 docking calculations performed, 47 ( $\sim .1 \%$ of all docking calculations) S-proteinACE2-ligand complexes were identified with scores equal to or better than the score threshold, and of these $27(\sim 0.05 \%)$ are reported (as noted in the ZINC15 database $\left.{ }^{36}\right)$ to be available for purchase. We selected for further analysis the top four poses of ligands already approved by regulators for use as either a drug or that have prior historical pharmaceutical uses, and their docking poses are represented in Figure 2.

The top four identified compounds are pemirolast ${ }^{37-38}$ (ZincID: 5783214), isoniazid pyruvate (ZincID: 4974291), nitrofurantoin (ZincID: 3875368), and eriodictyol (ZincID: 58117). Of the four small-molecules shown in figure 2, the top-ranked hit, pemirolast, is an anti-allergy medication or for use in treating chronic asthma ${ }^{37-38}$, while the second and third of the top hits are two are related to well-known antibiotics, with nitrofurantoin an antibiotic previously used for urinary tract infections ${ }^{39}$ and isoniazid pyruvate being a metabolite of the tuberculous 
antibiotic Isoniazid ${ }^{40}$. The last of the top four hits (Eriodictyol) is a flavanone found in Herba

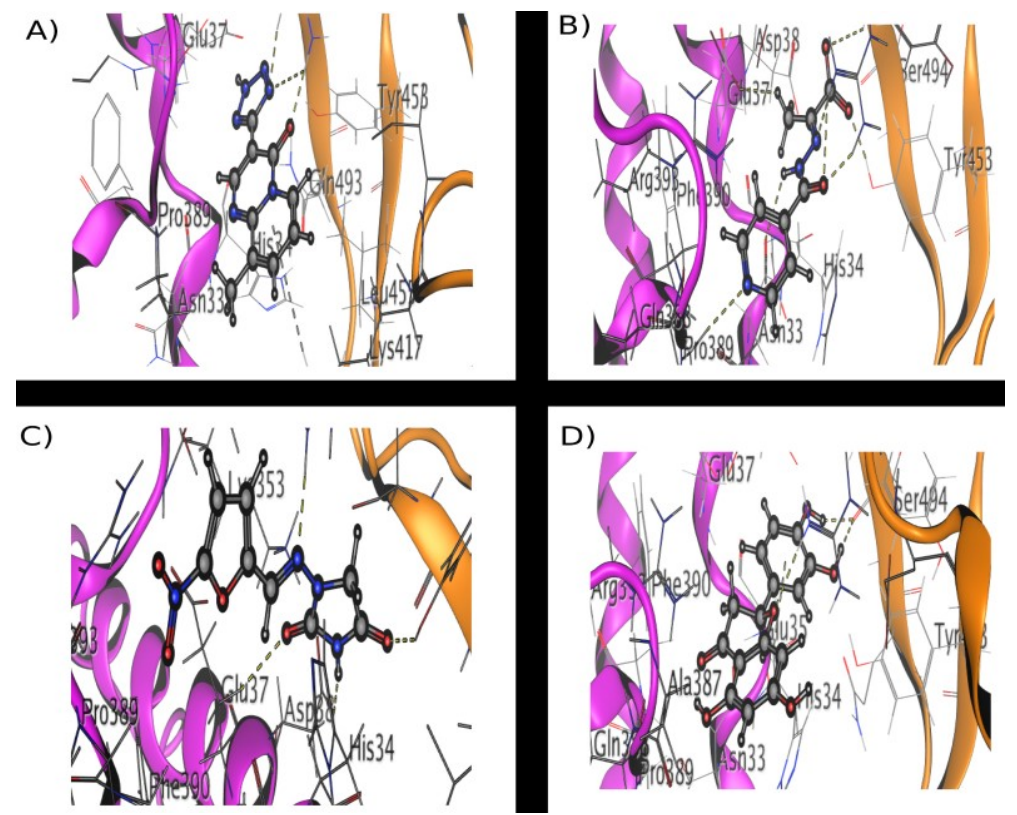

Figure 2) Renderings of four of the top scoring previously regulator approved small-molecules binding within the S-protein-ACE2 interface. A) pemirolast (ZincID: 5783214). B) isoniazid pyruvate (ZincID: 4974291). C) Nitrofurantoin (Zinc ID: 3875368). D) Eriodictyol (ZincID: 58117). Orange ribbons represent the S-protein and purple ribbons correspond to the ACE2 receptor

Santa, a traditional herbal remedy used for asthma and treating colds ${ }^{41}$.

Figures (3-6) provide a detailed two-dimensional accounting of the ligand-interface interactions. These interaction maps indicate that three of the four top hits (isoniazid pyruvate, nitrofurantoin, and eriodictyol) each contain nearly twice as many interactions with the ACE2 receptor than the S-protein, while pemirolast has roughly equal amounts of interactions between the two proteins. Further, the second through fourth top hits had 3 to 6 more ligand-residue interactions.

As the binding of the S-protein to ACE2 is undesirable, it is preferable to diminish ligandinterface interactions that may bridge, and therefore stabilize, the interaction between the Sprotein and the ACE2 receptor. 


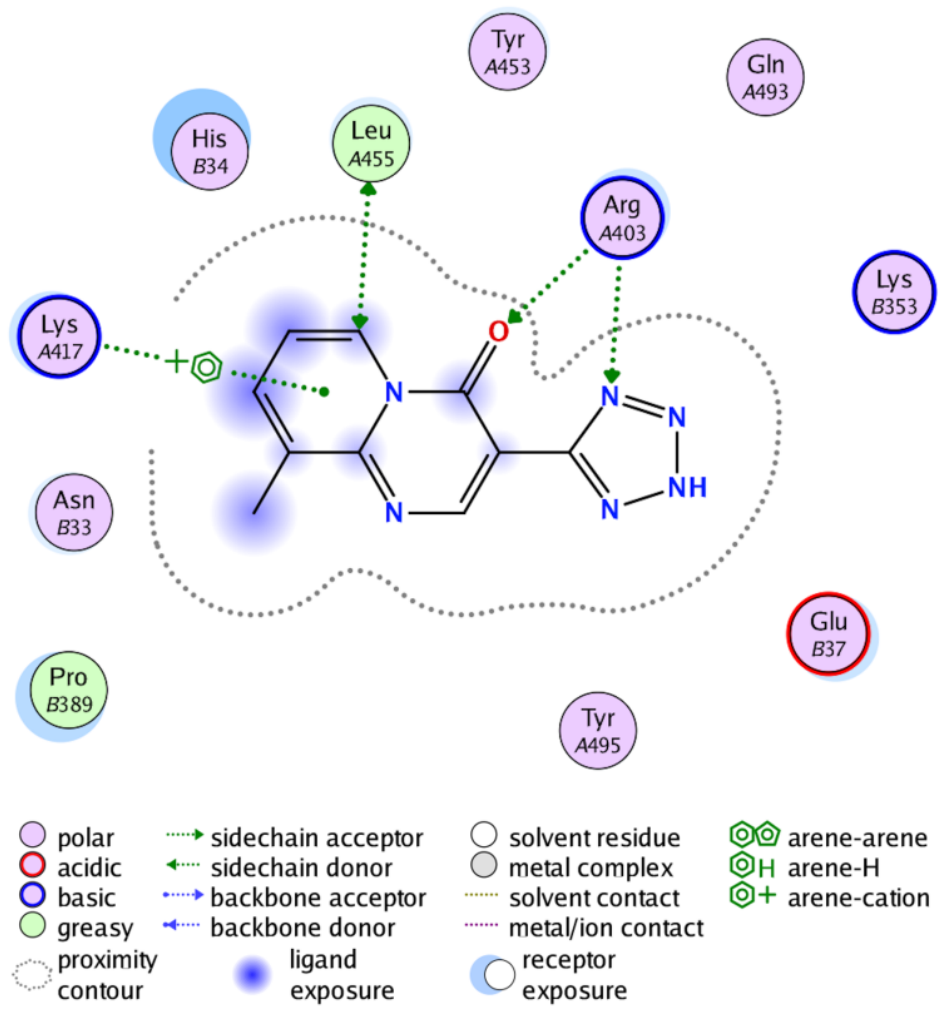

Figure 3) permirolast-protein Interface interaction diagram (generated from MOE2016). Residues with (A) correspond to the S-protein, while those with (B) correspond with the ACE2 receptor.

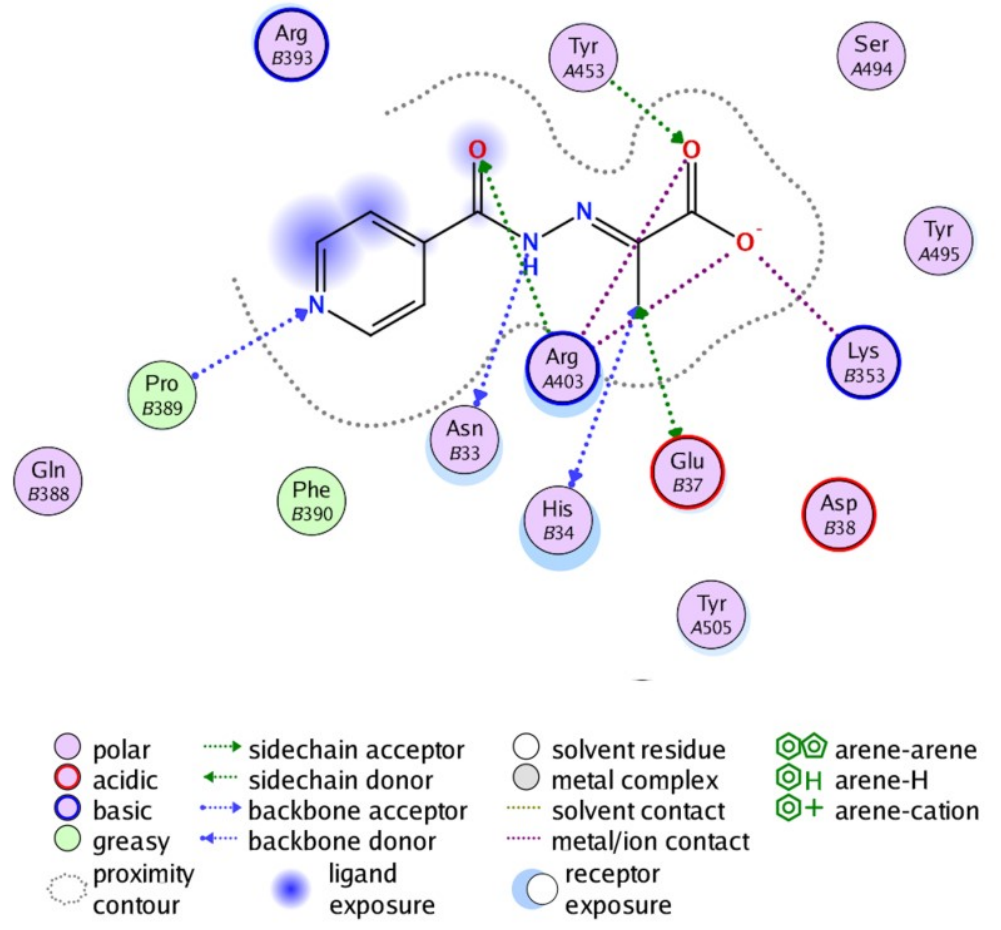

Figure 4) isoniazid pyruvate-protein Interface interaction diagram (generated from MOE2016). Residues with (A) correspond to the S-protein, while those with (B) correspond with the ACE2 receptor. 


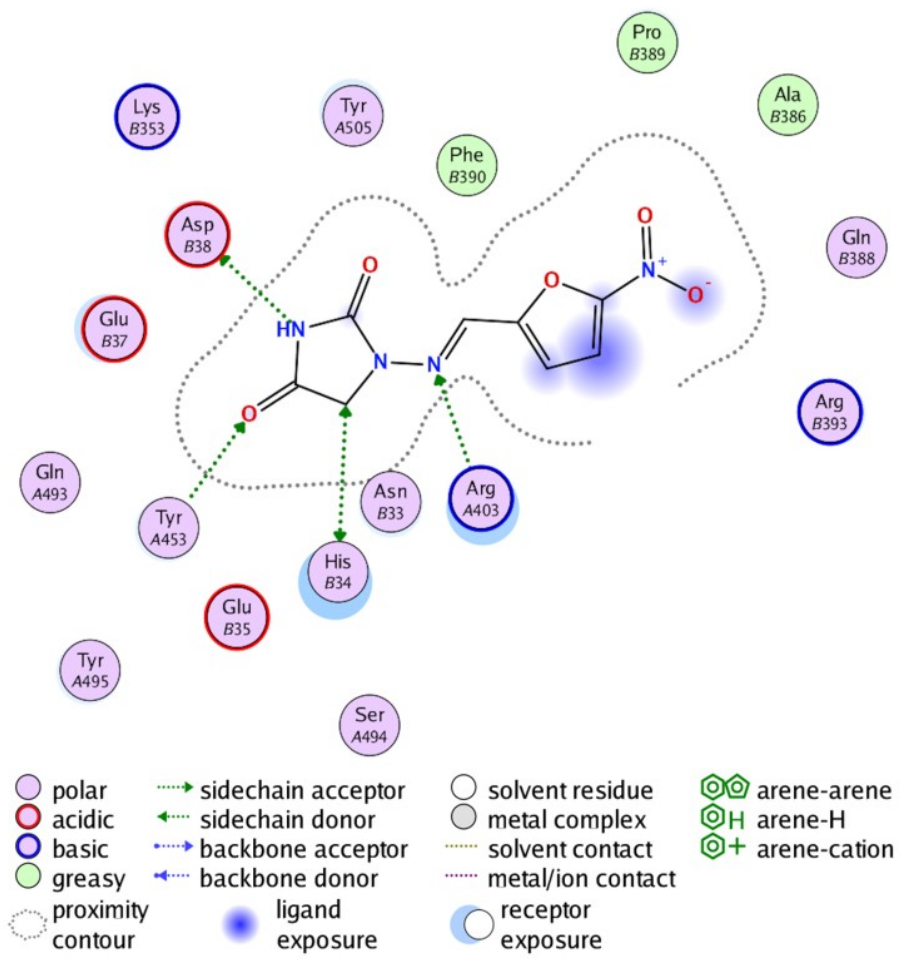

Figure 5) nitrofurantoin-protein Interface interaction diagram (generated from MOE2016). Residues with (A) correspond to the S-protein, while those with (B) correspond with the ACE2 receptor.

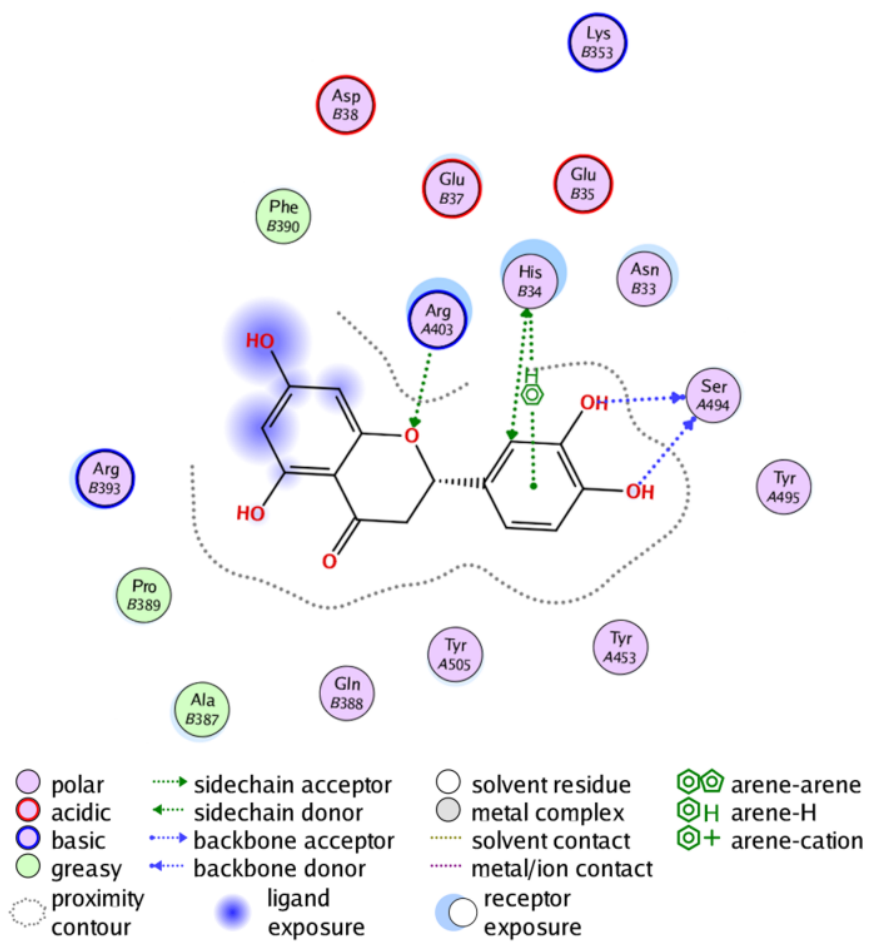

Figure 6) eriodictyol -protein Interface interaction diagram (generated from MOE2016). Residues with (A) correspond to the S-protein, while those with (B) correspond with the ACE2 receptor.

With this in mind, while the top hit, pemirolast, has the strongest binding affinity, initial 
repurposing may be better suited to isoniazid pyruvate, nitrofurantoin, or eriodictyol.

\section{Conclusion}

Prior work has demonstrated that $\mathrm{nCoV}-2019$ likely shares the ACE2 receptor as an entry point for infection with the SARS-CoV. Here we made use of enhanced sampling molecular simulations of currently available structure models of the S-protein of nCoV-2019 binding with the ACE2 receptor to generate an ensemble of configurations for ensemble docking. Further, we have made use of this ensemble to screen the SWEETLEAD library against the interface and have identified 47 potential hits, with 27 being currently available, which may favorably interact with the protein-protein interface. Of the 27 currently available, we highlighted four of our top compounds that have been previously used as drugs and as a potential drug. Of the four top compounds, three (nitrofurantoin, isoniazid pyruvate, and eriodictyol) were found to have a preference for residues belonging to the ACE2 receptor portion of the interface, and we hypothesize that these favorable interactions may in-turn limit the binding of the nCov-2019 Sprotein with the ACE2 receptor, thus restricting infection.

\section{Supplementary Information}

We provide for the interested reader a substantial portion of our raw data, including the PDB structures of the complex generated from SWISSMODEL, an excel spreadsheet containing the Vina score, internal ligand ID, ZINC-ID, and the commercial availability of the top 47 ligands identified by the docking calculations, Molecular Dynamics input files, and MD derived structural clusters. C code and accompanying bash-scripts used in this study to execute Vina over 600 SUMMIT nodes are also provided. Ligand-interface docking poses are available by request.

\section{Acknowledgments}


We acknowledge support from the Oak Ridge Leadership Computing Facility.

This manuscript has been authored by UT-Battelle, LLC under Contract No. DE-AC05-000R22725 with the U.S. Department of Energy. The United States Government retains and the publisher, by accepting the article for publication, acknowledges that the United States Government retains a non-exclusive, paid-up, irrevocable, world-wide license to publish or reproduce the published form of this manuscript, or allow others to do so, for United States Government purposes. The Department of Energy will provide public access to these results of federally sponsored research in accordance with the DOE Public Access Plan (http://energy.gov/downloads/doe-public-access-plan ).

\section{References}

1. Li, Q.-H.; Ma, Y.-H.; Wang, N.; Hu, Y.; Liu, Z.-Z., New Coronavirus-Infected Pneumonia Engulfs Wuhan. Asian Toxicology Research 2020, 1.

2. $\quad \mathrm{Xu}, \mathrm{X}$.; Chen, P.; Wang, J.; Feng, J.; Zhou, H.; Li, X.; Zhong, W.; Hao, P., Evolution of the novel coronavirus from the ongoing Wuhan outbreak and modeling of its spike protein for risk of human transmission. Science China Life Sciences 2020, 1-4.

3. Zhu, N.; Zhang, D.; Wang, W.; Li, X.; Yang, B.; Song, J.; Zhao, X.; Huang, B.; Shi, W.; Lu, R., A novel coronavirus from patients with pneumonia in China, 2019. New Engl J Med 2020.

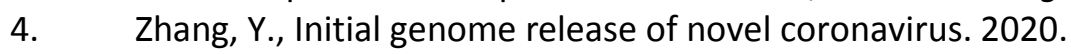

5. Lu, R.; Zhao, X.; Li, J.; Niu, P.; Yang, B.; Wu, H.; Wang, W.; Song, H.; Huang, B.; Zhu, N., Genomic characterisation and epidemiology of 2019 novel coronavirus: implications for virus origins and receptor binding. The Lancet 2020.

6. Wan, Y.; Shang, J.; Graham, R.; Baric, R. S.; Li, F., Receptor recognition by novel coronavirus from Wuhan: An analysis based on decade-long structural studies of SARS. Journal of Virology 2020.

7. Hoffmann, M.; Kleine-Weber, H.; Krüger, N.; Müller, M.; Drosten, C.; Pöhlmann, S., The novel coronavirus 2019 (2019-nCoV) uses the SARS-coronavirus receptor ACE2 and the cellular protease TMPRSS2 for entry into target cells. bioRxiv 2020, 2020.01.31.929042.

8. Wang, C.; Horby, P. W.; Hayden, F. G.; Gao, G. F., A novel coronavirus outbreak of global health concern. The Lancet 2020.

9. $\quad$ Amaro, R. E.; Baudry, J.; Chodera, J.; Demir, Ö.; McCammon, J. A.; Miao, Y.; Smith, J. C., Ensemble docking in drug discovery. Biophys $J$ 2018, 114 (10), 2271-2278.

10. Huang, S. Y.; Zou, X., Ensemble docking of multiple protein structures: considering protein structural variations in molecular docking. Proteins: Structure, Function, and Bioinformatics 2007, 66 (2), 399-421.

11. Pi, M.; Kapoor, K.; Wu, Y.; Ye, R.; Senogles, S. E.; Nishimoto, S. K.; Hwang, D.-J.; Miller, D. D.; Narayanan, R.; Smith, J. C., Structural and functional evidence for testosterone activation of GPRC6A in peripheral tissues. Mol Endocrinol 2015, 29 (12), 1759-1773.

12. Pi, M.; Kapoor, K.; Ye, R.; Nishimoto, S. K.; Smith, J. C.; Baudry, J.; Quarles, L. D., Evidence for osteocalcin binding and activation of GPRC6A in $\beta$-cells. Endocrinology 2016, 157 (5), 1866-1880.

13. Evangelista, W.; Weir, R. L.; Ellingson, S. R.; Harris, J. B.; Kapoor, K.; Smith, J. C.; Baudry, J., Ensemble-based docking: From hit discovery to metabolism and toxicity predictions. Bioorgan Med Chem 2016, 24 (20), 4928-4935.

14. Abdali, N.; Parks, J. M.; Haynes, K. M.; Chaney, J. L.; Green, A. T.; Wolloscheck, D.; Walker, J. K.; Rybenkov, V. V.; Baudry, J.; Smith, J. C., Reviving antibiotics: efflux pump inhibitors that interact with AcrA, a membrane fusion protein of the AcrAB-TolC multidrug efflux pump. ACS infectious diseases 2017, 3 (1), 89-98. 
15. Haynes, K. M.; Abdali, N.; Jhawar, V.; Zgurskaya, H. I.; Parks, J. M.; Green, A. T.; Baudry, J.; Rybenkov, V. V.; Smith, J. C.; Walker, J. K., Identification and Structure-Activity Relationships of Novel Compounds that Potentiate the Activities of Antibiotics in Escherichia coli. Journal of medicinal chemistry 2017, 60 (14), 6205-6219.

16. Velazquez, H. A.; Riccardi, D.; Xiao, Z.; Quarles, L. D.; Yates, C. R.; Baudry, J.; Smith, J. C., Ensemble docking to difficult targets in early-stage drug discovery: Methodology and application to fibroblast growth factor 23. Chemical biology \& drug design 2018, 91 (2), 491-504.

17. Darzynkiewicz, Z. M.; Green, A. T.; Abdali, N.; Hazel, A.; Fulton, R. L.; Kimball, J.; Gryczynski, Z.; Gumbart, J. C.; Parks, J. M.; Smith, J. C., Identification of binding sites for efflux pump inhibitors of the AcrAB-TolC component AcrA. Biophys J 2019, 116 (4), 648-658.

18. Pi, M.; Kapoor, K.; Ye, R.; Smith, J. C.; Baudry, J.; Quarles, L. D., GPCR6A is a molecular target for the natural products gallate and EGCG in green tea. Molecular nutrition \& food research 2018, 62 (8), 1700770.

19. Xiao, Z.; Baudry, J.; Cao, L.; Huang, J.; Chen, H.; Yates, C. R.; Li, W.; Dong, B.; Waters, C. M.; Smith, J. C., Polycystin-1 interacts with TAZ to stimulate osteoblastogenesis and inhibit adipogenesis. The Journal of clinical investigation 2018, 128 (1), 157-174.

20. Biasini, M.; Bienert, S.; Waterhouse, A.; Arnold, K.; Studer, G.; Schmidt, T.; Kiefer, F.; Cassarino, T. G.; Bertoni, M.; Bordoli, L., SWISS-MODEL: modelling protein tertiary and quaternary structure using evolutionary information. Nucleic Acids Res 2014, 42 (W1), W252-W258.

21. Schwede, T.; Kopp, J.; Guex, N.; Peitsch, M. C., SWISS-MODEL: an automated protein homologymodeling server. Nucleic Acids Res 2003, 31 (13), 3381-3385.

22. Bjelkmar, P.; Larsson, P.; Cuendet, M. A.; Hess, B.; Lindahl, E., Implementation of the CHARMM Force Field in GROMACS: Analysis of Protein Stability Effects from Correction Maps, Virtual Interaction Sites, and Water Models. J Chem Theory Comput 2010, 6 (2), 459-466.

23. Van der Spoel, D.; Lindahl, E.; Hess, B.; Groenhof, G.; Mark, A. E.; Berendsen, H. J. C., GROMACS: Fast, flexible, and free. J Comput Chem 2005, 26 (16), 1701-1718.

24. Huang, J.; MacKerell Jr, A. D., CHARMM36 all-atom additive protein force field: Validation based on comparison to NMR data. J Comput Chem 2013, 34 (25), 2135-2145.

25. Berendsen, H. J. C.; Postma, J. P. M.; Vangunsteren, W. F.; Dinola, A.; Haak, J. R., MolecularDynamics with Coupling to an External Bath. J Chem Phys 1984, 81 (8), 3684-3690.

26. Abraham, M. J.; Gready, J. E., Optimization of Parameters for Molecular Dynamics Simulation Using Smooth Particle-Mesh Ewald in GROMACS 4.5. J Comput Chem 2011, 32 (9), 2031-2040.

27. Hess, B.; Bekker, H.; Berendsen, H. J. C.; Fraaije, J. G. E. M., LINCS: A linear constraint solver for molecular simulations. J Comput Chem 1997, 18 (12), 1463-1472.

28. Hess, B., P-LINCS: A parallel linear constraint solver for molecular simulation. J Chem Theory Comput 2008, 4 (1), 116-122.

29. Bussi, G.; Donadio, D.; Parrinello, M., Canonical sampling through velocity rescaling. J Chem Phys 2007, $126(1)$.

30. Daura, X.; Gademann, K.; Jaun, B.; Seebach, D.; Van Gunsteren, W. F.; Mark, A. E., Peptide folding: when simulation meets experiment. Angewandte Chemie International Edition 1999, 38 (1-2), 236-240.

31. Huey, R.; Morris, G. M., Using AutoDock 4 with AutoDocktools: a tutorial. The Scripps Research Institute, USA 2008, 54-56.

32. Novick, P. A.; Ortiz, O. F.; Poelman, J.; Abdulhay, A. Y.; Pande, V. S., SWEETLEAD: an in silico database of approved drugs, regulated chemicals, and herbal isolates for computer-aided drug discovery. Plos One 2013, 8 (11).

33. Inc., C. C. G., Molecular operating environment (MOE). Chemical Computing Group Inc 1010 Sherbooke St. West, Suite\# 910, Montreal: 2016. 
34. Trott, O.; Olson, A. J., AutoDock Vina: improving the speed and accuracy of docking with a new scoring function, efficient optimization, and multithreading. J Comput Chem 2010, 31 (2), 455-461.

35. Halgren, T. A., Merck molecular force field. I. Basis, form, scope, parameterization, and performance of MMFF94. J Comput Chem 1996, 17 (5-6), 490-519.

36. Sterling, T.; Irwin, J. J., ZINC 15-ligand discovery for everyone. J Chem Inf Model 2015, 55 (11), 2324-2337.

37. Kemp, J.; Bernstein, I.; Bierman, C.; Li, J.; Siegel, S.; Spangenberg, R.; Tinkelman, D., Pemirolast, a new oral nonbronchodilator drug for chronic asthma. Ann Allergy 1992, 68 (6), 488-491.

38. Fujimiya, H.; Nakashima, S.; Miyata, H.; Nozawa, Y., Effect of a novel antiallergic drug, pemirolast, on activation of rat peritoneal mast cells: inhibition of exocytotic response and membrane phospholipid turnover. International Archives of Allergy and Immunology 1991, 96 (1), 62-67.

39. D'Arcy, P. F., Nitrofurantoin. Drug intelligence \& clinical pharmacy 1985, 19 (7-8), 540-547.

40. Timmins, G. S.; Deretic, V., Mechanisms of action of isoniazid. Molecular microbiology 2006, 62 (5), 1220-1227.

41. Ley, J. P.; Krammer, G.; Reinders, G.; Gatfield, I. L.; Bertram, H.-J., Evaluation of Bitter Masking Flavanones from Herba Santa (Eriodictyon californicum (H. \& A.) Torr., Hydrophyllaceae). J Agr Food Chem 2005, 53 (15), 6061-6066. 